\title{
OPTIMIZED CONFIGURATIONS FOR MICRO-PHOTOGRAMMETRIC SURVEYING ADAPTABLE TO MACRO OPTICS AND DIGITAL MICROSCOPE
}

\author{
S. Antinozzi ${ }^{1}$, F. Fiorillo ${ }^{2}$
}

' Dept. of Civil Engineering (DICIV), Università degli Studi di Salerno, Italy - santinozzi@unisa.it

2Dept. of Architecture, Built environment and Construction engineering (DABC), Politecnico di Milano, Italy - fausta.fiorillo@polimi.it

\author{
Commission II
}

KEY WORDS: Small Artefacts, Digital Heritage, Micro Survey, Detail Scale, Handheld Microscope, Image-based Modelling.

\begin{abstract}
:
The configuration of new survey approaches for digitizing tiny artefacts is gradually emerging in favour of the related results sharing in new modalities such as 3D printing and or Cultural Heritage open-access datasets. Unfortunately, due to the still high cost of the instrumentations and the time required for specialized operators training, both the digital documentation of small artefacts and the dissemination of 3D data are still a long way from becoming truly mainstream practices.

Alternative solutions, often at low cost, could be considered, involving the use of passive sensors for image-based modelling processes, relying on a widespread technology that is now within everyone's reach. Therefore, we intend to meet the demand for the threedimensional representation of small objects with complex surfaces and sub-millimetre morphological characteristics by designing several custom accessories that optimize the photogrammetric workflow while maintaining very high metric rigour.

In this current work, the authors will focus on researching the most suitable acquisition method and hardware setup to achieve a digital twin of a complex and detailed cuneiform tablet replica using the now widely available on the market digital portable microscopes, able to achieve high magnification. The results were compared with a reference dataset from an active sensor acquisition for the necessary procedure validations.
\end{abstract}

\section{INTRODUCTION}

The Cultural Heritage digital documentation techniques are well defined for dimensions and architectural graphic representation scales but still not conveniently formalized and codified for very tiny objects. Interfacing with small finds represents a complex challenge due to the level of detail change and the need to update/re-think the measurements operating systems. Nevertheless, the $3 \mathrm{D}$ reconstruction of small objects is becoming increasingly widespread in various fields, especially the manufacturing industry, mechanics, medical sciences, including also archaeological documentation, interested in using emerging and available digital methodologies and technologies provided by Geomatics, and aiming at rigorous objects description (Bitelli et al., 2007).

Several solutions for micrometric surveying applications (Hansen et al., 2006), including range-based ones (Tolksdorf et al., 2017), or integrated approaches - still representing the most effective solution (Morena et al., 2019) - are increasingly succeeding in the task.

Unfortunately, most of these technologies are out of reach of most museums and cultural institutions due to the considerable hardware and software costs and the level of expertise required of the operators (Caine and Maggen, 2017). Today's market demand for practical, low-cost solutions directs the interest towards more affordable alternatives, such as microphotogrammetry. This solution offers the possibility of obtaining three-dimensional coordinates of an object and, at the same time, the exact corresponding radiometric information from two-dimensional digital images in an accurate, reliable, flexible, and, often, money-saving way.

The issue is even more interesting considering the demand for technologically advanced experiences we are currently used to when visiting museums, art galleries or archaeological sites.

All these factors open new frontiers to digital photogrammetry for renewing the use of Cultural Heritage (Mancuso and
Pasquali, 2015). In addition, such discipline is open to further technological developments and implementations, such as speeding up the acquisition phase. Today this first step represents its weak point because it is difficult, repetitive and time-consuming for operators.

Starting from this awareness, our current challenge is configuring a highly performative micro-photogrammetric system designed for both: i) micro-photogrammetry most established solutions, based on digital camera combined with macro lenses; ii) and also portable digital microscopes, whose validity has been tested for micro-scale survey also in our previous works.

Thus, this system aims to overcome some of the operational difficulties experienced in recent applications and to contain the problems that affect very close-range photogrammetry, such as not very high resolution, lack of a wide dynamic range, narrow field of view and poor depth of field. Indeed most of the problems that arise - despite the use of increasingly advanced software for photogrammetric processing - are actually generated by purely photographic errors or by a lack of knowledge of photographic tools (Verdiani et al., 2018).

Comparing two procedural solutions - simultaneously evaluating the development of the accurate and cost-effective micro-photogrammetric acquisition system - the authors propose to carry out the digital survey of a 3D printing replica in geopolymer of a tablet with cuneiform writings. This case study has approximately the dimensions of $20 \times 22 \times 8 \mathrm{~mm}$, and the characteristic wedge-shaped impressions have a depth of 1-3 $\mathrm{mm}$ (Figure 1). Tablets inscribed with the cuneiform script were the 'portable information technology' of that time; in this case, the text content concerns the administrative information for fish deliveries (the modern correspondent of a packing slip).

The replica, used as a test, is a printed clone of the original 724 tablet (realized by the $+\mathrm{LAB}$, www.piulab.it - Politecnico di Milano) surveyed using an active sensor system by the 3D Survey Group (Politecnico di Milano). 


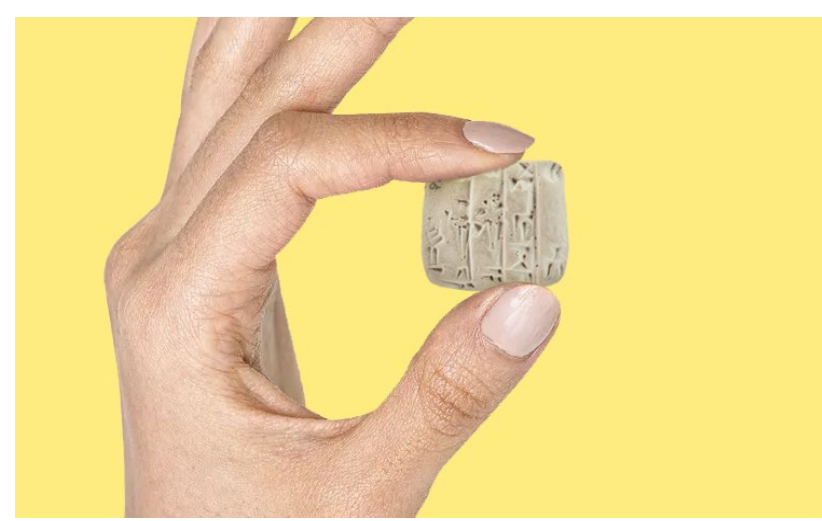

Figure 1. Recto of the 3D printed copy of the cuneiform tablet.

It should be noted that original tablets are easily susceptible to damage, and scholars have limited tools for accessing and recording these finds (Surdi, 2016; Lewis and Ch'ng, 2012).

The success of the conducted tests on this copy can ensure that in the future, a digital clone with high accuracy of original cuneiform tablets - or even other similar artefacts with great tiny detail - can be quickly and lows cost obtained for educational and scientific purposes. The digital replica would allow the virtual manipulation of these delicate findings, and facilitate their sharing among a team of archaeologists too, for example, to decipher the content of a text, thus favouring an interdisciplinary study among several experts.

\section{RELATED WORKS}

Close-range photogrammetry involves many parameters that affect the acquisition process, and this is compounded by increasing difficulties as the scale of application reduces.

One of the main problems is the need to include metric references or calibrated objects in the scene, using adequate calibration patterns according to the magnitude of the object to be measured (Lavecchia et al., 2017).

Another critical issue concerns the acquisition geometry, which must ensure sufficient overlap between the captured images to reconstruct the object surface and its feature continuity. Furthermore, while turntables facilitate and speed up the acquisition, there are very few possibilities of housing the object in an advantageous position to ensure the necessary base between the camera positions.

Finally, it should not be overlooked that the short depth of field issue is emphasized when increasing magnification. This aspect implies that only a tiny portion of the artefact is in focus on the photo, and so only a small portion of the image appears sharp enough to be used for 3D reconstruction (Clini et al., 2016). Indeed the sharpness, joint with photoset density and resolution, determines the output point cloud reliability, accuracy and quality (Westoby et al., 2012). These limitations could affect both the dataset acquisition and processing phases reciprocally. Indeed to ensure the suitable number of point matching and conjugate points computed and the resolution of the 3D model (Kontogianni et al., 2017), the survey times increase because it is necessary to move the camera often to cover the whole object surface. In order to overcome these difficulties, the authors conducted some experiments in previous studies to digital survey very tiny and problematic artefacts by optimizing the acquisition procedures with the design of custom accessories for micro-photogrammetry. Using photographs taken by low-cost Dino-Lite (www.dinolite.eu) digital microscopes (Figure 2) as a dataset for photogrammetric processing, a not negligible potential for small

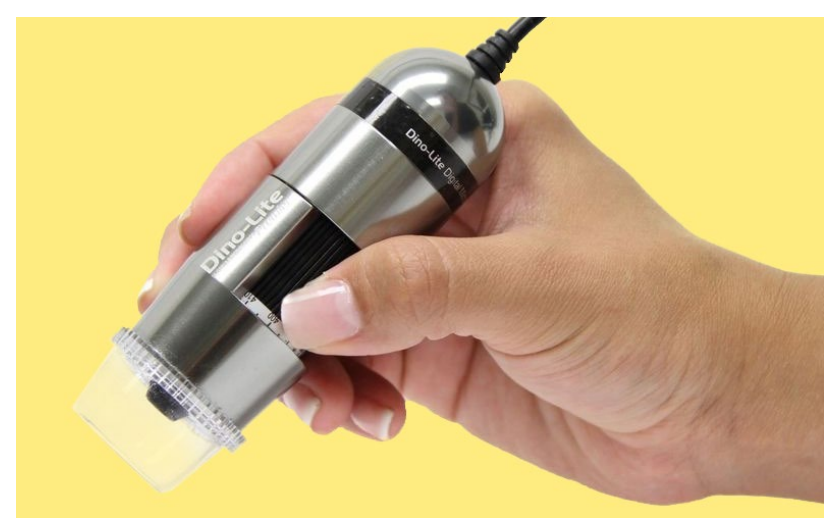

Figure 2. Dino-Lite handheld digital microscope.

object modelling - compared with significantly more expensive instruments - was revealed (Antinozzi et al., 2021a).

As is well known, digital handheld microscopes are similar to traditional optical microscopes, except that they are equipped with a CCD camera to output a digital colour image to a monitor. Moreover, among the main types of microscopes, they are the most flexible, the easiest to use, and the least expensive (Atsushi et al., 2011). Born for inspection, documentation, and digital metrology analysis - already widespread in the manufacturing and quality control industry and used in the medical field - digital microscopes can be used for microphotogrammetric applications, reaching about $0,1 \mathrm{~mm}$ accuracy (Esmaeili and Ebadi, 2017). It may seem interesting, therefore, to insert these instruments within a systematized photogrammetric workflow. Their main disadvantage is that they are not born purely for photogrammetric purposes and therefore need to be adapted, for example, through the design of a specific calibrator - to guarantee accurate results - and of an automatic system that speeds up the data acquisition. Furthermore, for the same reason, no technical calibration characteristics of the lens system are known.

\section{MATERIALS AND METHODS}

\subsection{An original asset for the micro-photogrammetry}

The previous tests conducted by the authors to achieve a digital twin of a complex and detailed 3D printing replica of a tablet with cuneiform writing, as challenging as the original, are valid for defining a first approach to the photogrammetric use of portable digital microscopes (Antinozzi et al., 2021b).

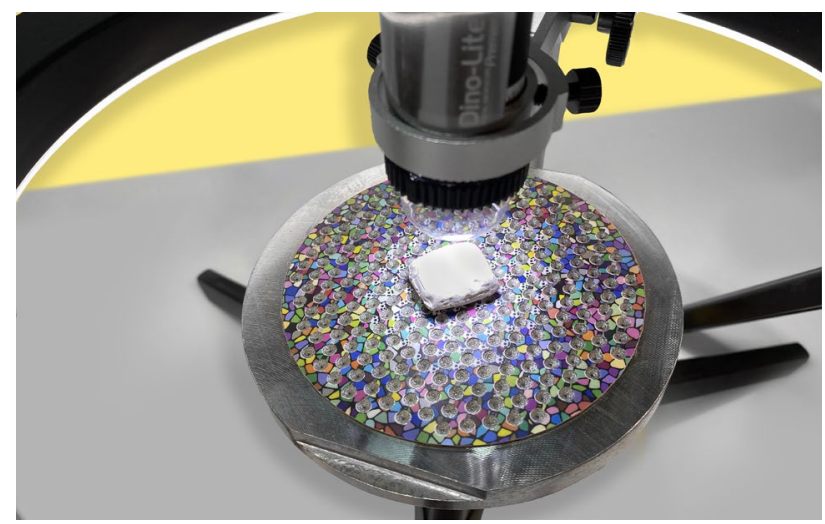

Figure 3. Three-dimensional calibrated plate combined with the digital microscope (updated prototype). 


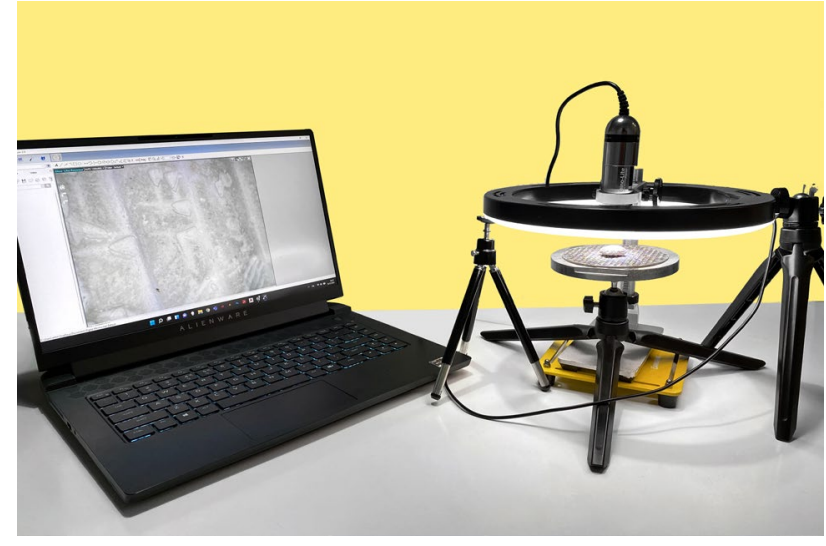

Figure 4. Setup configuration for the first acquisition test, included calibrator, its support and lighting system.

Nevertheless, on this occasion, some key aspects are still more rigorously investigated to streamline the acquisition procedures and ensure a more rigorous workflow.

A redesign of the acquisition hardware for this dimensional scale is required to allow you to have everything you would need in one single system for the photo modelling of tiny objects. It implies: calibrated stands to hold the object and at the same time ensure the metric accuracy, the rotating base to help you and speed up acquisitions, the lighting suitable for each situation. For this particular application, having already experimented with using a flat calibrated support, it was decided in a first step to implement the characteristics of the old calibrated plate by designing a new one. Subsequently, an alternative solution was tested, evaluating the possibility of using an adhesive pattern glued onto clamp support. Even in this second mode, therefore, it would have been possible to frame the metric references and the object at the same time, with the hope of further reducing surveying times.

Hence, first, the authors designed a calibrated plate built for these tests (Figure 3) and used it for the standard photogrammetric procedure needs (images alignment, self-calibration optimization, and scale). This calibrator is obtained with an EOS M270, via additive manufacturing of steel powder and consists of a circular plate characterized by a pattern of 279 truncated cone holes, with a countersink angle of $60^{\circ}$ and a smaller base diameter of 0.35 $\mathrm{mm}$. The calibrator accuracy, based on the $3 \mathrm{D}$ printing settings and the conformity of the actual position of the holes with respect to the project file, has been estimated at $0,1 \mathrm{~mm}$, which can be assumed as an instrumental error value (i.e., markers accuracy). Therefore, each hole coordinates in a local reference system are known to the operator and can be imported into the project.

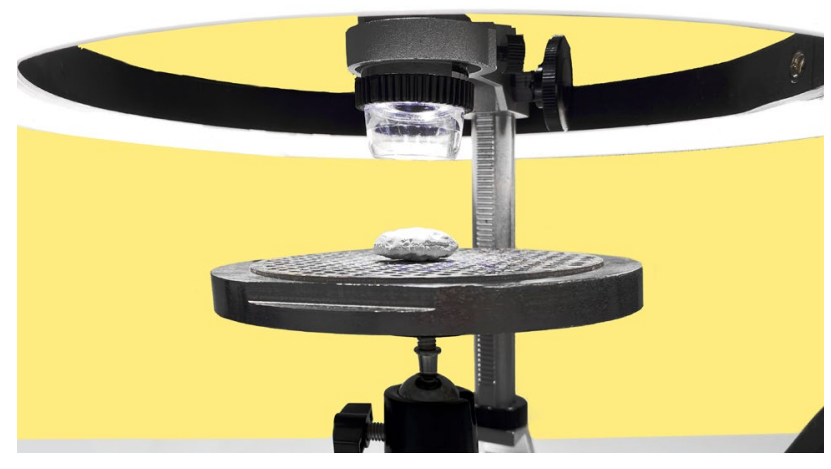

Figure 5. Detail of the lighting ring coaxial to the rotating base, on which the calibrated plate is mounted.
For this reason, the perforated pattern can be used as a constraint points (GCP) grid - homogeneously distributed throughout the whole area - to optimize the alignment of the cameras and scale the model. The calibrated plate was set on a turning table to facilitate and speed up the acquisition phase.

Once the magnification rate (optical zoom) has been chosen and placed the digital microscope on a fixed bracket at a fixed inclination, a set of images with sufficient overlap can be acquired by rotating the base. The angle of the plate to the camera is changed after each full rotation (Figure 4 and Figure 5).

The AM7013MZT Dino-Lite digital microscope was employed for this application. It is characterized by a 5-megapixel sensor for crystal clear images and a built-in adjustable polariser to reduce glare and reflection on shiny objects. This model offers a 'standard' working distance, as defined by the manufacturer, meaning a distance from the object that varies approximately from 50 to $2 \mathrm{~mm}$ : in our case, with a magnification rate of 20x, the focus is obtained at a working distance of $48.7 \mathrm{~mm}$, with a depth of field equal to $3.6 \mathrm{~mm}$.

In general, to overcome the problem of the short depth of field, one possibility would be to close the aperture of the optics as much as possible within the limits of the diffraction phenomena (Sapirstein, 2018) and provide enough light to balance a correct exposure time. Since this possibility is not available on portable digital microscopes, where the diaphragm is fixed, and consequently, the sharp field range is also fixed, it is necessary to use alternative solutions. For this purpose, the object is acquired from various focus planes by moving the microscope on the micrometric vertical rail to focus on different planes.

The lighting conditions, based on the polarised light of the microscopes, have been improved with the adoption of a LED illumination ring (Figure 5). The light does not directly affect the object because of the shape and the lens hood diffusing light source material; thus, diffused light conditions neutralize the shadow cones without variations in the intensity of shadows, lights, and colours.

With this system, two acquisition sets - one for the recto and the other for the verso of the tablet - averaging 250 captures each were required, for a total of about 1 hour of time needed.

One of the problems left open for the cuneiform tablet digital survey (and therefore for similar small objects with a not negligible thickness) is the impossibility to have the joined $3 \mathrm{D}$ model of the two faces (recto and verso) using the microscope dataset, although inclined acquisitions have been made. This particular and not trivial circumstance has forced us to take a further step forward. Having selected an object that, for its size and complexity, allows to stress the acquisition system and bring out its weaknesses, we understood how to deal with this critical issue by maintaining a low-cost approach.

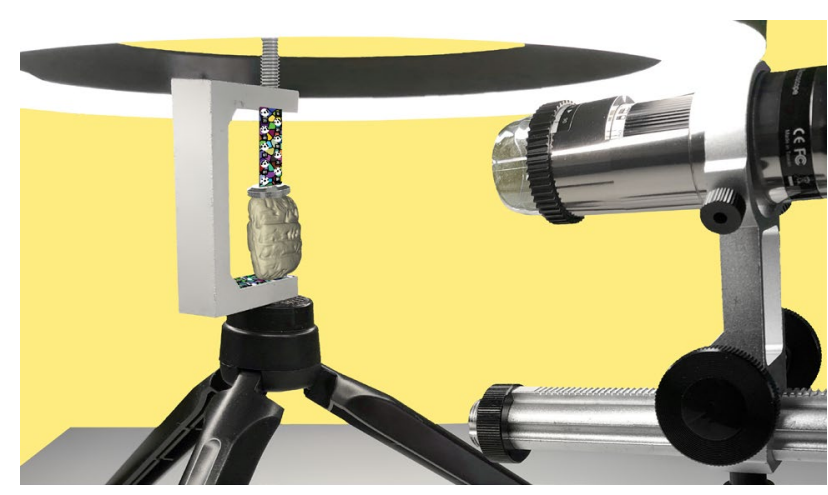

Figure 6. Setup of the second acquisition test, using the screw clamp coaxial to the rotating base. 


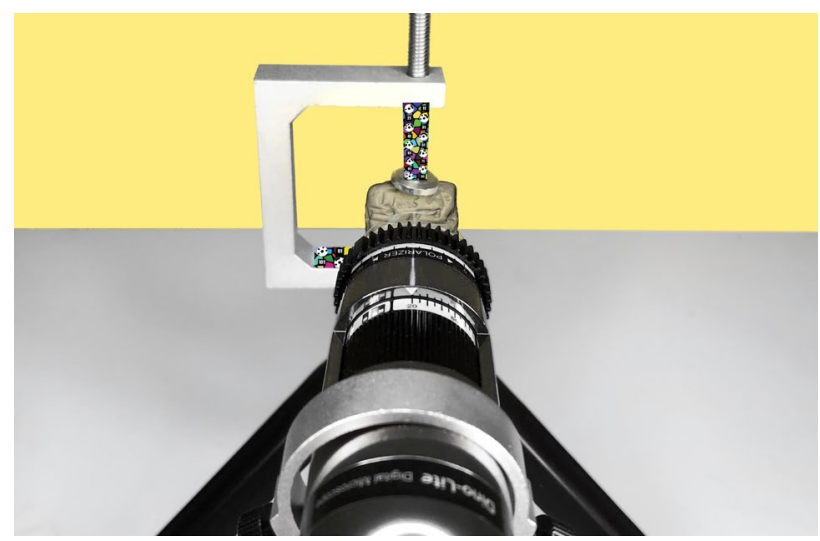

Figure 7. Detail of the positioning of the tablet in the clamp for the second acquisition test.

We have come, therefore, to conceive the possible modification of the object support (Figure 6), incorporating the calibrator in it. In this way, the surveyor has the possibility to use the same shooting system, choosing, if necessary, the support that is best suited to fix the object to be detected. The plate would, thus, be mainly suited only when it can be brought into sharp focus, even with objects of significant 3D shape (in the case of cameras where adjustment of the diaphragm of the optical apparatus allows the depth of field to be increased).Conversely, such as in our case, a screw clamp (Figure 7), commonly used in model making, would be more convenient to acquire objects to be placed at knife-edge in reference to the microscope camera. This acquisition mode enables the calibrated pattern to be positioned approximately coplanar to the plane of the object's focus (Figure 8). Easily available, the clamp was coated with an adhesive pattern on which automatically recognized targets of known coordinates were printed. Similarly to the calibrated plate, the clamp was also placed on a rotating base, taking care to align the rotation centre with the mounting axis of the object. Other photogrammetric accessories remain almost unchanged when compared to the previous accessories setup.

A set of about 700 captures was required with this system, and a supplement set for the small parts hidden by the clamp (about 100 captures), for a total of about 1 hour required.

For both mounting systems used, the acquisitions were carried out with the same magnifications, 20x, operating with the dedicated DinoCapture 2.0 software procedure (www.dinolite.eu/index.php/it/software-dino-lite).

The datasets obtained were thus processed in a SfM software, Agisoft Metashape, according to the general photogrammetric workflow (Table 1) and the processing was completed with the same graphic workstation equipped with AMD Ryzen 9 5900HX CPU and 32GB of RAM.

\begin{tabular}{|c|c|c|}
\hline $\begin{array}{c}\text { Acquisition } \\
\text { Geometry }\end{array}$ & $\begin{array}{c}\text { Calibrated Plate } \\
\text { Set }\end{array}$ & $\begin{array}{c}\text { Screw Clamp } \\
\text { Set }\end{array}$ \\
\hline Aligned Cameras & $506 / 518$ & $718 / 718$ \\
Sparse Cloud & $4.3 \times 10^{5}$ points & $4.1 \times 10^{5}$ points \\
Sparse Cloud Filtered & $3.8 \times 10^{5}$ points & $2.1 \times 10^{5}$ points \\
Dense Cloud & $10.4 \times 10^{5}$ points & $8.3 \times 10^{5}$ points \\
Ground Resolution & $6.3 \mu \mathrm{m} / \mathrm{px}$ & $7.14 \mu \mathrm{m} / \mathrm{px}$ \\
RMS Error & $0.87 \mathrm{~mm}$ & $0.027 \mathrm{~mm}$ \\
Processing Time & $4 \mathrm{~h}$ & $2 \mathrm{~h}$ \\
& & \\
\hline
\end{tabular}

Table 1. Survey data comparison.

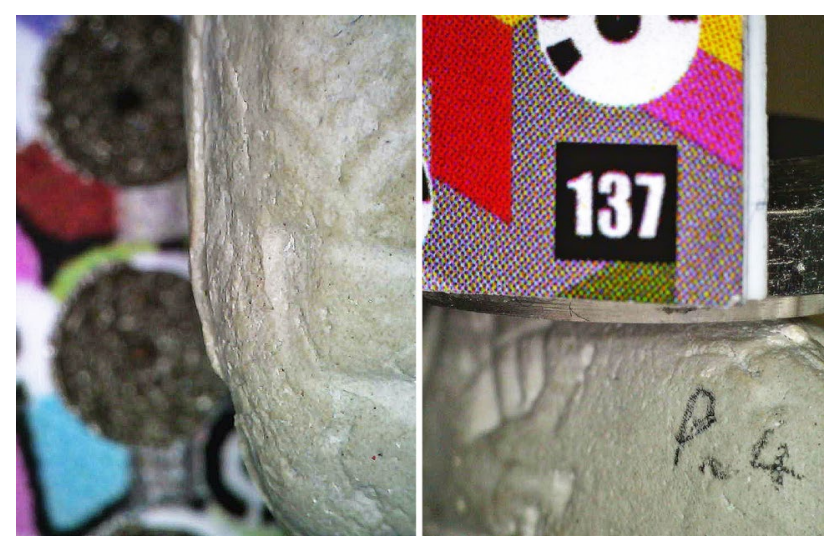

Figure 8. Different ability to focus on target and object at the same time using the calibrated plate (left) or the clamp (right).

During the processing of the first test, there is a significant difficulty in aligning tilted photos due to the shallow depth of field and the homogeneous texture of the tablet.

This problem was not encountered in the second test because the capture geometry forced (and facilitated) the operator to take more photos due to the particular location of the object with respect to the camera (Figure 9).

The sparse point cloud, made only by tie points (TPs), is the starting point for the realization of a complete $3 \mathrm{D}$ model. However, removing low-quality TPs is appropriate because their presence affects the following step results, which consist of the re-computation of the orientation parameters and the creation of the final dense cloud (Barba et al., 2019).

The evaluation of image orientation quality within SfM methods can be performed using the Gradual Selection filter, a tool by Agisoft Metashape that, like most photogrammetric software, allows you to filter the sparse point cloud based on some quality parameters. The parameters considered in this study were: Reconstruction uncertainty, Reprojection error and Projection accuracy.

\subsection{Reference model equipment}

The quality, speed, and cost-effectiveness of the two surveys conducted with the portable Dino-Lite digital microscope were compared to data obtained from the SCAN in a BOX structured light 3D scanner to have a reference model and check the overall geometrical dimensions of the photogrammetric reconstructions.

The SCAN in a BOX (@2015 Open Technologies SRL) is equipped with two high-resolution industrial USB cameras and a high definition light projector (ASUS S1).
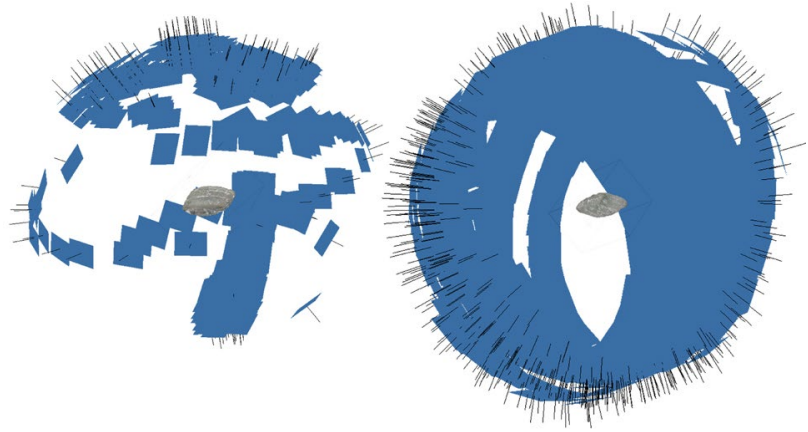

Figure 9. Camera spatial positions during the calibrated plate (left) and screw clamp acquisition test (same time taken: $1 \mathrm{~h}$ ). 


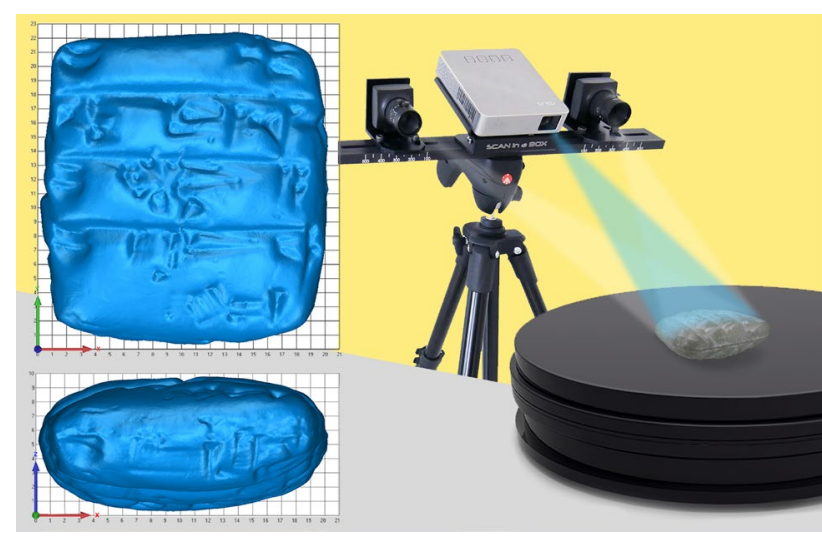

Figure 10. "SCAN in a BOX" system and polygonal model of the tablet acquired ( $1 \mathrm{~mm}$ grid background).

All components are fixed on a thick aluminium rail, but the cameras can be moved in calibrated positions (base) to customize the work field (Figure 10). The main steps for the 3D measurements workflow are the following: i) calibration of the optical setup, ii) range maps acquisition, iii) raw alignment, iv) alignment optimization and v) meshing. The system needs to be configured and calibrated whenever the scanner is mounted, or the camera setting on the support bar changes. The coded calibration master has three patterns based on the selected working area.

The optical setup provides the configuration of the projector and cameras (position, orientation, focus, exposition).

The calibration allows calculating the optical parameters of the specific setting. According to the object dimension and the resolution required, choosing the scanning area is more suitable, strictly connected to the working distance and the distance of the cameras on the plate.

For our case study, a Field Of View of 100 x $80 \mathrm{~mm}$ was set, implying the working distance scanner-object of about $200 \mathrm{~mm}$, the minimum base between the two cameras, and the minimum point spacing (resolution) on the surface, i.e. $0.078 \mathrm{~mm}$.

The digital reconstruction of the object surface and details was performed, acquiring 16 nadiral scans on both sides $(8$ for the verso and 8 for the recto), 16 tilted ( 8 for each side) and 8 nadiral to the four edges (totally of 40 range maps). The scanning time for each range map is less or equal to 4 seconds. The raw in progress alignment process helped check real-time scan completeness. This initial registration is then optimized using an Iterative Closest Point (ICP) algorithm.

The final mesh model (formed by about 400.000 polygons) have fewer details of the correspondent photogrammetric models but can be used to ensure a reference check of their global measures.

\section{EVALUATION OF THE RESULTS}

This analysis aims to evaluate the photogrammetric models obtained from the two different procedures illustrated: the first based on the use of a calibrated plate on which to place the object being surveyed, the second on the use of a clamp in which to secure the object and a portion of which is covered by a calibration pattern.

This is to draw some first conclusions on the efficiency and accuracy capabilities of the two different acquisition methods.

A cloud to mesh registration has been performed using the data from the SCAN in a BOX as a reference to provide a robust comparison of the results.
The registration was carried out in two steps, providing: first, a manual registration using homologous points and then a global registration by automatic alignment algorithms (ICP). The photogrammetric survey accuracy assessment was carried out using CloudCompare C2M cloud to mesh) command. This tool searches, for each point being compared, the closer reference entity, thus defining a shift value of the first with respect to the second. The clouds obtained from photogrammetry were compared using the SCAN in a BOX model as a reference.

Figure 11 shows the deviations among the clouds in false colours. Through the calculations performed it is noted that the mean and standard deviations from the reference do not exceed $0,3 \mathrm{~mm}$ for both photogrammetric models. This difference could have been determined by both the registration procedure and the higher resolution obtained from the photogrammetric survey.

Indeed the SCAN in a BOX mesh model is smoother and has less resolution (about $0.08 \mathrm{~mm}$ ).

The results obtained using the calibrated plate are noticeably affected by greater deviations, due on the one hand to image alignment problems, which have also significantly increased the noise of the dense cloud, and on the other hand to the difficulty of joining the two sides of the tablet. This comparison suggests that the shape of our object requires different capture sets, with different inclinations of the microscope, probably much more than those made by rotating the object placed in the clamp. Therefore, the manual acquisition will be more challenging to consider a corresponding increase in processing time due to the need to perform the same photogrammetric workflow for each acquisition set and merge the different blocks.

Therefore, we consider the result of the first test satisfactory both metrically and for an overall reading of the artefact, but the threedimensional model, excessively noisy and needing interventions a posteriori, is not excellent for a complete user experience. However, it is believed that the calibrated plate remains a valuable tool when used for mostly flat objects and/or combined with SLR cameras and macro lenses, allowing for greater depth of field and wider angle of view. As acknowledged, in fact, a generally applicable geometric configuration for photogrammetric measurement cannot be defined (Luhmann et al., 2011) as this depends on the specific object to be detected. Nevertheless, having several alternative supports available allows the capture geometry to be quickly modified. Although also in the second test, it was necessary to turn the piece over and partially repeat the capture process for the occluded part, in order to achieve full digitization of the tablet, the results obtained using the clamp are very satisfactory.

In the post-processing phase, it was expeditious to align the partial scans and create a single 3D mesh of the complete object. For this second model, approximately $94 \%$ of the points are included in a smaller difference than $+/-0.1 \mathrm{~mm}$, versus around $40 \%$ of the previous (Figure 12). Noise is very low, limited only to certain portions of the object, and the final model is readable and complete, lending itself to wide dissemination: https://skfb.ly/o8GSr.

The two models comparison (Figure 13) highlights in detail that the model generated with the calibrated plate setup has problems both along the edges and in some parts of the main faces, with unnatural bulbs on the surface due to excessive noise.

Indeed, in this case, the sparse cloud filtering - performed to detect and remove outliers and mismatches affecting the image orientation results - would have caused excessive data loss, returning equally noisy but severely incomplete three-dimensional reconstructions.

In addition, in both cases, the uncertainty generated by the absence of Exif data must be considered as a further detrimental parameter for the alignment processes and the resulting issues. 

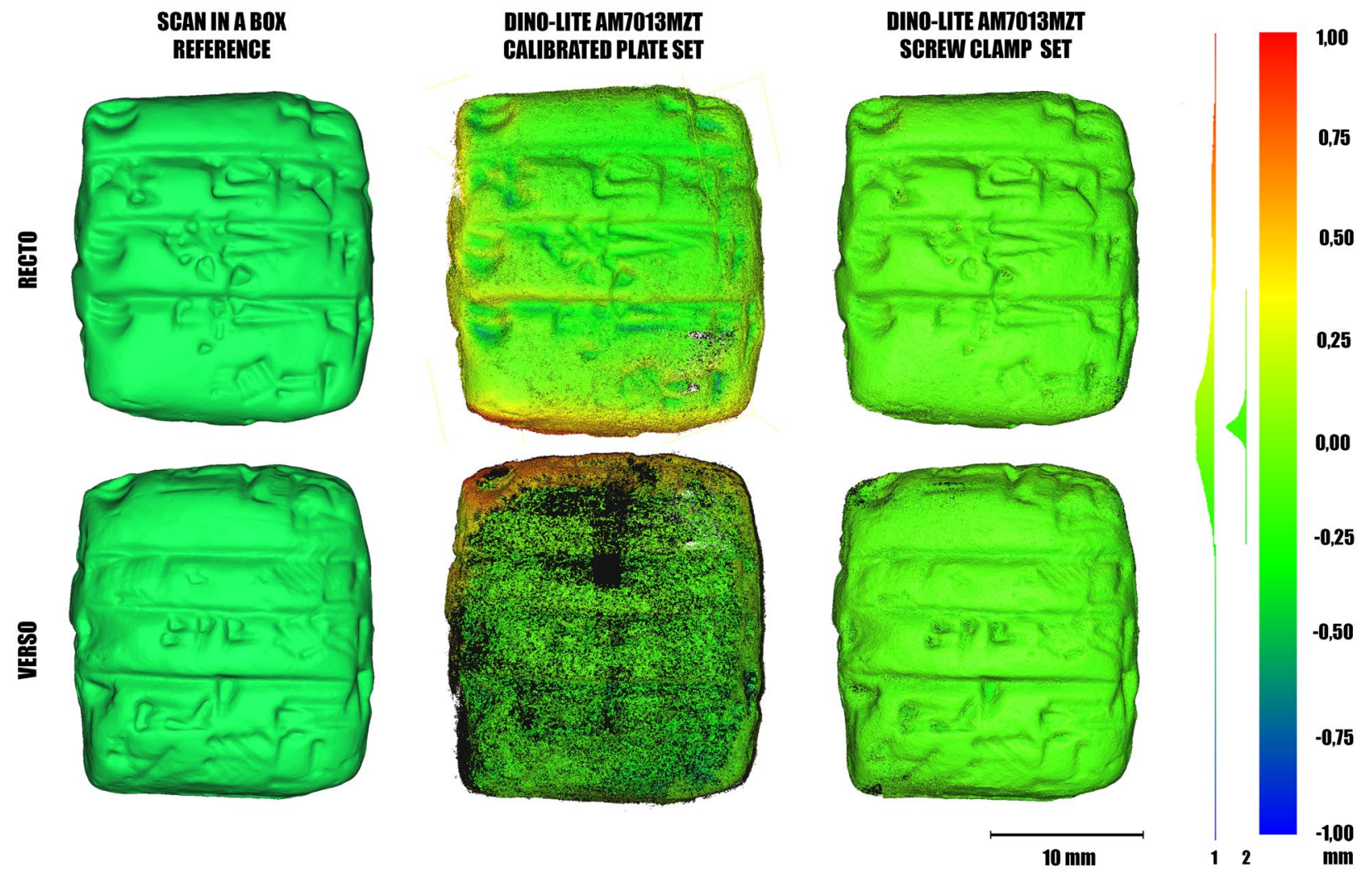

Figure 11. Global deviations of the photogrammetric dense clouds obtained using the calibrated plate and the screw clamp as support, compared with the reference model of the SCAN in a BOX (recto and verso side view).

\section{DINO-LITE AM7013MZT CALIBRATED PLATE SET}

C2M signed distances $[<1]$

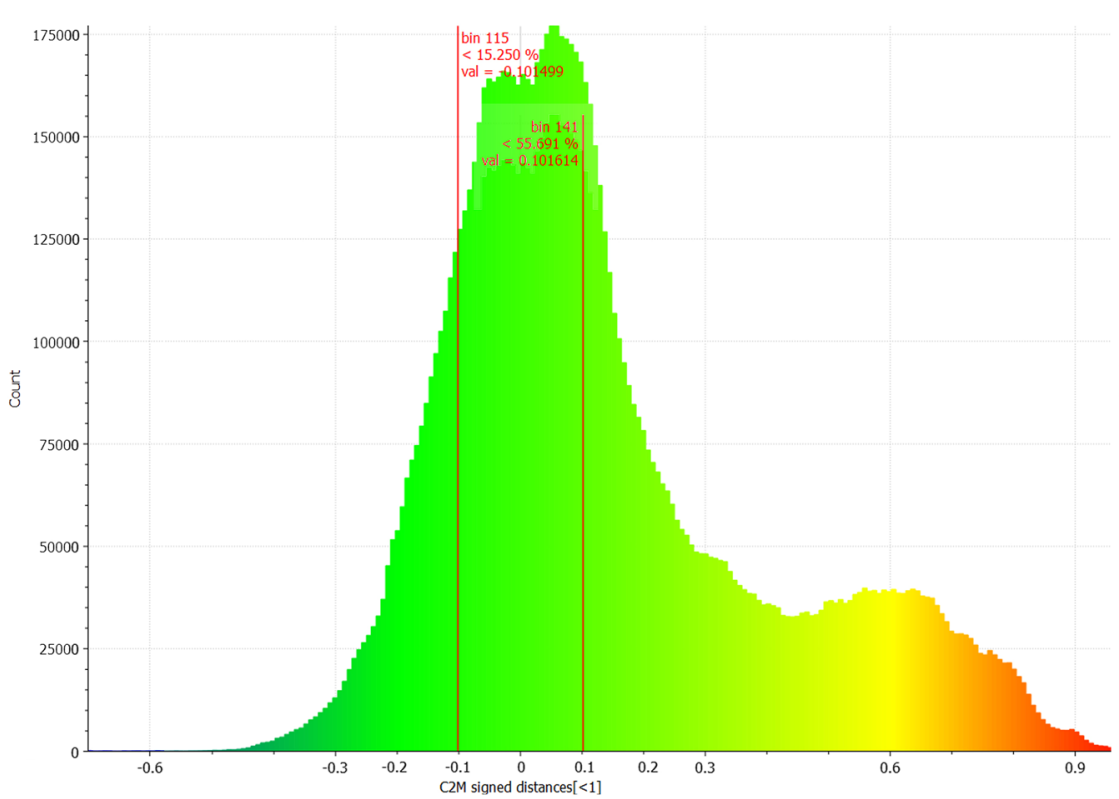

DINO-LITE AM7013MZT SEREW GLAMP SET

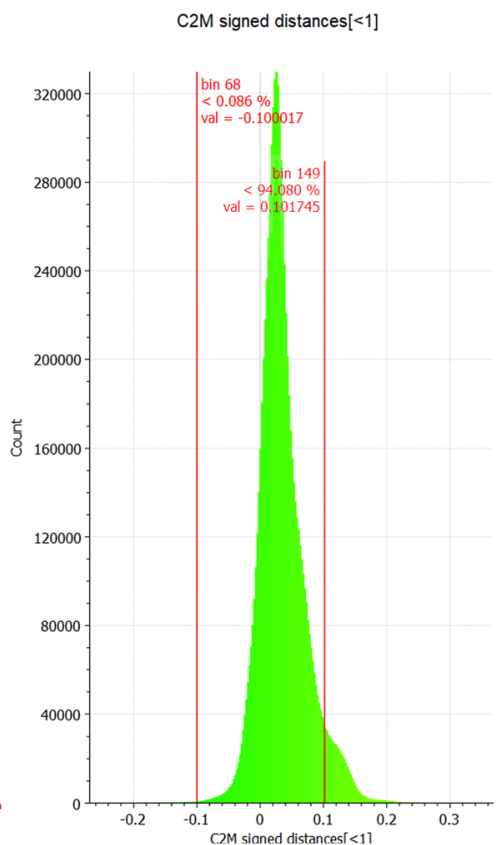

Figure 12. Global distribution detailed chart of photogrammetric cloud deviations from the reference model: in the central portion of the graphic, approximately $40 \%$ (left) and $94 \%$ (right) of the points are included in a difference of $+/-0.1 \mathrm{~mm}$. 


\section{GALIBRATED PLATE SET VERTIGES: 1.048.050, FACES: 2.090 .368}
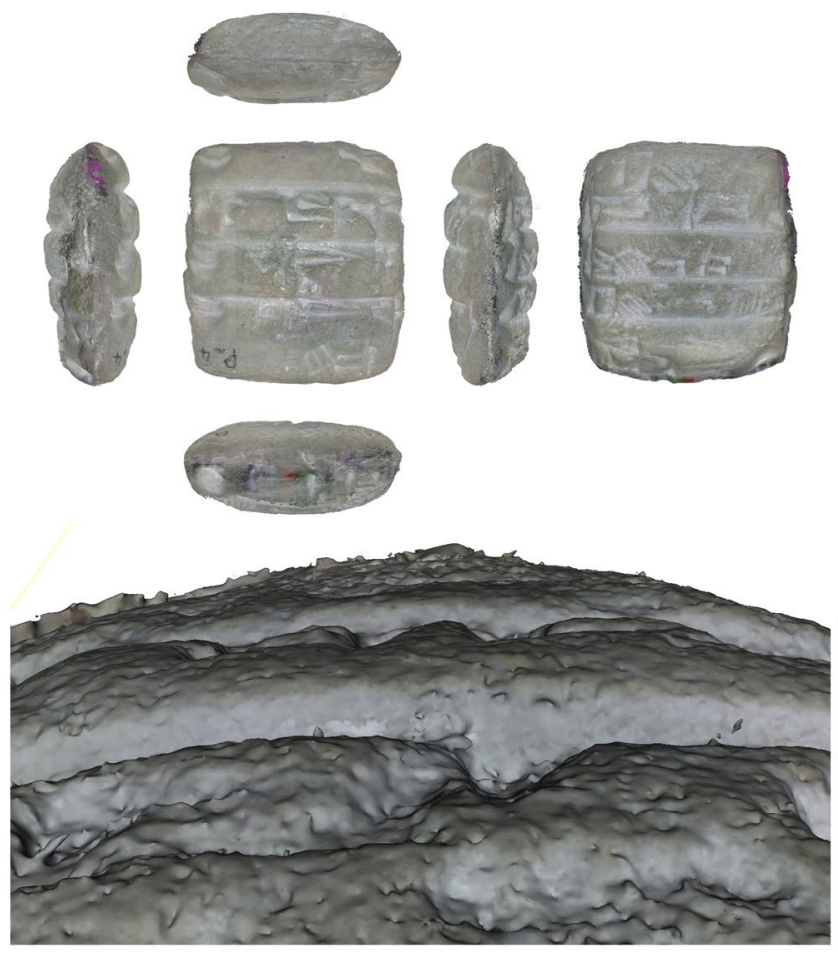

Figure 13. Qualitative comparison of the models obtained using the calibrated plate (left) and the screw clamp as support (right).

\section{SCREW CLAMP SET VERTIGES: 833.503, FACES: 1.666 .834}
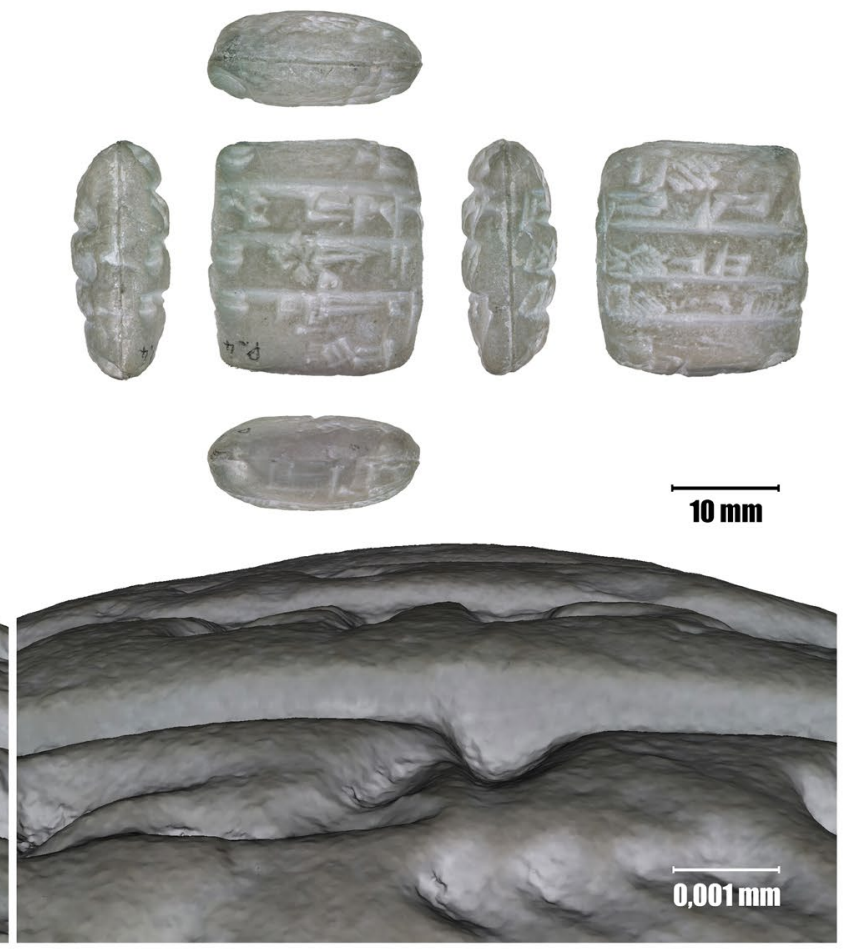

Despite the problems within the framework of modern microtechnologies, the results from Dino-Lite are absolutely comparable in shape and size to the SCAN in a BOX, ensuring a slightly higher resolution and thus demonstrating that digital microscopes combined with an optimized setup can be effective and interesting tools for micro-photogrammetry.

\section{FUTURE DEVELOPMENTS}

Having access to a low-cost system based on the principles of close-range photogrammetry, at the same time efficient and affordable, is, therefore, a still unmet need for those who want to share and circulate digital models of objects with particular reference to the scale of detail. In this sense, in order to meet this demand, there are still several key issues to be resolved not as mere executors but as proponents of new tools and technologies. Indeed, each object represents a uniqueness for which it is necessary to design a custom survey plan. Nevertheless, today it is possible to realize, at quite low costs, different interchangeable supports, equipped with calibrated patterns, in order to fix the object in the best way, to neutralize the shadow cones at the most and to guarantee the metric accuracy, which is the primary aim of the survey. Therefore, the main purpose of this research in the future will focus on implementing the design of three-dimensional calibrators to support the 3D reconstruction of tiny objects with a not negligible thickness. This will be followed by the characterization of the other necessary components, including, for example, the diffuse annular illumination, the support for the optical system and the rotating base, which at this stage have been combined in a still 'rudimentary' way.
These latest experimental tests conducted with the Dino-Lite instruments are valid to take a further step forward in an attempt to combine the various elements and solutions that make up a complete photogrammetric system, optimized for acquisition and ensuring the control of the subsequent management of the measurement data.

Furthermore, there are interesting ideas for the three-dimensional documentation of very small artefacts that would otherwise be difficult to represent from this work in progress. Indeed, additional tests are already planned using more resolute and accurate active sensors to verify further the results achieved. In this way, it would be possible to replicate at low cost and with adequate accuracy the work of instrumentation that exceeds the cost of these microscopes with a relatively low effort.

\section{ACKNOWLEDGEMENTS}

We would like to thank, sincerely, IDCP Digital Innovation - in the persons of Jan Boers, Danielle van Duijvendijk and Ivo Manders - and, of course, Dino-Lite Digital Microscope for the support, availability and equipment offered for the research. Special thanks to Diego Ronchi (ISPC, Consiglio Nazionale delle Ricerche), Mirko Surdi (Ghent University), Catherine Mittermayer (Department of Antiquity Sciences, University of Geneva), Francesco Fassi (3D Survey Group, Politecnico di Milano) and Marinella Levi (+LAB, Politecnico di Milano) for sharing dataset, materials and researches. 


\section{REFERENCES}

Antinozzi, S., Ronchi, D., Barba, S., 2021a. 3Dino System, Shortening Distances in Precision Surveys. In A. Arena, M. Arena, D. Mediati and P. Raffa (eds.), Connecting. Drawing for weaving relationship. Languages Distance. FrancoAngeli, Milano, 1922-1941.

Antinozzi, S., Ronchi, D., Fiorillo, F., Barba, S., 2021b. 3Dino: configuration for a micro-photogrammetric survey. Applying Dino-Lite microscope for the digitalization of a cuneiform tablet. Proceedings of the 39th eCAADe Conference - Towards a new, configurable architecture. Novi Sad, Serbia, September 8-10, 2, 211-222.

Atsushi, K., Sueyasu, H., Funayama, Y., Maekawa, T., 2011. System for reconstruction of three-dimensional micro objects from multiple photographic images. Computer-Aided Design, 43, $8,1045-1055$

Barba, S., Barbarella, M., Di Benedetto, A., Fiani, M. Limongiello, M., 2019. Comparison of uavs performance for a roman amphitheatre survey: The case of Avella (Italy). ISPRS International Archives of the Photogrammetry, Remote Sensing and Spatial Information Sciences, 3XLII-2/W11, 179-186.

Bitelli, G., Girelli, V. A., Remondino, F., Vittuari, L., 2007. The potential of 3D techniques for Cultural Heritage object documentation. Proceedings of Videometrics IX - SPIE-IS\&T Electronic Imaging, San Jose, California, USA, January 29February 1, 64910S, 244-253.

Caine, M. and Maggen, M., 2017. Low Cost Heritage Imaging Techniques Compared. Proceedings of the Conference on Electronic Visualisation and the Arts, London, UK, July 11-13, 430-437.

Clini, P., Frapiccini, N., Mengoni, M., Nespeca, R., Ruggeri, L., 2016. SFM Technique and Focus Stacking for Digital Documentation of Archaeological Artifacts. ISPRS International Archives of the Photogrammetry, Remote Sensing and Spatial Information Sciences, XLI-B5, 229-236.

Esmaeli, F. and Ebadi, H., 2017. Handy Microscopic CloseRange Videogrammetry. ISPRS International Archives of the Photogrammetry, Remote Sensing and Spatial Information Sciences, XLII-4/W4, 65-67.

Hansen, H., Carneiro, K., Haitjema, H., De Chiffre, L., 2006. Dimensional Micro and Nano Metrology. Cirp Annals Manufacturing Technology, 55, 2, 721-743.

Kontogianni, G., Chliverou, R., Koutsoudis, A., Pavlidis, G., Georgopoulos, A., 2017. Enhancing close-up image based 3D digitization with focus stacking. ISPRS International Archives of the Photogrammetry, Remote Sensing and Spatial Information Sciences, XLII-2/W5, 421-425.

Lavecchia, F., Guerra, M. G., Galatucci, L. M., 2017. Performance verification of a photogrammetric scanning system. The International Journal of Advanced Manufacturing Technology, 96, 4267-4279.

Lewis, A. and Ch'ng, E., 2012. A Photogrammetric Analysis of Cuneiform Tablets for the purpose of Digital Reconstruction. International Journal of Cultural Heritage in the Digital Era, 1, Suppl 1, 49-54.
Luhmann, T., Robson, S., Kyle, S., Harley, I., 2011. Close Range Photogrammetry. Principles, techniques and applications. Whittles Publishing, Dunbeath, Scotland, UK, 443.

Mancuso, A. and Pasquali A., 2015. Digital MicroPhotogrammetry. New ways to dialogue with future researchers. Proceedings of the 20th International Conference on Cultural Heritage and New Technologies 2015, Vienna, Austria, November 10-12, 1-16.

Morena, S., Barba, S., Álvaro-Tordesillas, A., 2019. Shining 3D Einscan-Pro, application and validation in the field of Cultural Heritage, from the Chillida-Leku Museum to the Archaeological Museum of Sarno. ISPRS International Archives of the Photogrammetry, Remote Sensing and Spatial Information Sciences, XLII-2/W18, 135-142.

Sapirstein, P., 2018. A high-precision photogrammetric recording system for small artifacts. Journal of Cultural Heritage, 31, 3345.

Surdi, M., 2017. Dalla tavoletta al tablet: metodi classici e nuove tecnologie a confronto per l'Assiriologia. Archeomatica, 7, 4, 4046 .

Tolksdorf, J. F., Elburg, R., Reuter, T., 2017. Can 3D scanning of countermarks on Roman coins help to reconstruct the movement of Varus and his legions. Journal of Archaeological Science: Reports, 11, 400-410.

Verdiani, G., Formaglini, P., Giansanti, F., Giraudeau, S., 2018. Close-Up, Macro and Micro-photogrammetry and Image Perspective: A Comparative Studio on Different Lenses at Work with Small and Medium Size Objects. Computer Reviews Journal, 2, 235-248.

Westoby, M., Brasington, J., Glasser, N., Hambrey, M., Reynolds, J., 2012. Structure-from-Motion photogrammetry: A low-cost, effective tool for geoscience applications. Geomorphology, 179, 300-314. 